\title{
On The Design of Two-Channel 2-D Nonseparable Multi-Plet Perfect Reconstruction Filter Banks
}

\author{
K. M. Tsui and S. C. Chan
}

Department of Electrical and Electronic Engineering,

The University of Hong Kong, Pokfulam Road, Hong Kong.

\begin{abstract}
This paper proposes a new design method for a class of two-channel 2D non-separable perfect reconstruction (PR) filter banks (FBs) using the multi-plet FBs. 1D multi-plet FBs are PR FBs that can be obtained by frequency transformation of a prototype $\mathrm{PR}$ FB in the conventional lifting structure so that a better frequency characteristics can be obtained and varied online to process different signals. By employing the $1 \mathrm{D}$ to $2 \mathrm{D}$ transformation of Phoong et al, new 2D PR multi-plet FBs with quincunx, hourglass, and parallelogram spectral support are obtained. These nonseparable multi-plet FBs can be cascaded to realize new $\mathrm{PR}$ directional $\mathrm{FB}$ for image processing and motion analysis. The design procedure is very general and it can be applied to both linear-phase and low-delay 2D FBs. Design examples are given to demonstrate the usefulness of the proposed method.
\end{abstract}

\section{INTRODUCTION}

Perfect reconstruction (PR) filter banks (FBs) have important applications in signal analysis, coding and the design of wavelet bases. An efficient structure of two-channel biorthogonal FIR/IIR FBs, which structurally satisfies the PR condition, is the structural PR FB proposed by Phoong et al. [1]. Moreover, the FBs of this structure can be transformed, via a simple $1 D$ to $2 \mathrm{D}$ transformation, to obtain 2D PR FIR nonseparable filter banks with quincunx spectral support. One limitation of this structure is that the magnitudes of the lowpass and highpass analysis filters at $\omega=\pi / 2$ in the linear-phase case are respectively restricted to 0.5 and 1 , or vice versa. In another structural PR FBs called triplet FBs [2] - [4], a generalization of the structure in [1], more degree of freedom is available and it is possible to achieve a more symmetric frequency response. More recently, Chan et al. extended the structural PR FBs [1] and the triplet FBs [2] - [4], which involve two and three lifting steps [5], respectively, and studied a new class of two-channel structural PR FBs with multiple lifting steps called the multiplet FBs [6]. In particular, they showed that the concept of frequency transformation of digital filters studied in [7] can be applied directly to the lifting structure to obtain another PR FB with the same number of lifting steps having the similar frequency characteristics but an arbitrary sharp transition bandwidth. This can also be viewed as an extension of the work in [8].

In this paper, we shall extend the $1 \mathrm{D}$ to $2 \mathrm{D}$ transformation of Phoong et al. [1] to the multi-plet FBs. The design of such PR 2D nonseparable FBs can be divided into three steps. Firstly, a low order prototype PR FB with a rather wide transition bandwidth is first designed to meet certain specifications on passband and stopband ripples and it is then factorized into lifting structure [9]. Alternatively, the well known lifting structured FB, say $(9,7)$ filter pair [10], can be used. Secondly, by properly designing the subfilter of transformation, the frequency response of the prototype FB can be warped by means of frequency transformation to meet the desired transition bandwidth, while preserving the PR condition and the frequency characteristics of the prototype FB. Also, the lengths and the passband delays of the analysis/synthesis filters can be made closer to each other, which simplifies practical implementation and reduces the number of additional delay elements required for processing two subband signals. Finally, similar to the $1 \mathrm{D}$ to $2 \mathrm{D}$ mapping used in [1] and [11], the 1D multi-plet FB can be readily transformed to construct $2 \mathrm{D} P R$ FB with quincunx, hourglass and parallelogram spectral supports. Note that these multi-plet 2D nonseparable PR FBs can also be implemented as a lifting structure with the same number of lifting steps as its prototype. Furthermore, they can be cascaded appropriately in a tree-structure to obtain new PR directional FBs, using the approach previously proposed in [12]. Such directional FBs found important applications in segmentation, directional decomposition of images, motion analysis of videos, etc [13]. Design examples are given to illustrate the potential flexibility of the proposed approaches in processing $1 \mathrm{D}$ and $2 \mathrm{D}$ signals.

The paper is organized as follows: the 1D multi-plet FBs and the concept of frequency transformation are introduced in section II. Their generalizations to 2D nonseparable PR FBs with quincunx, hourglass and parallelogram spectral supports are discussed in Section III. Several design examples are given in section IV to illustrate the effectiveness of the proposed approach, and finally, conclusion is drawn in section V.

\section{Multi-Plet Two-Channel Structural PR FBs}

The general structure of the multi-plet two-channel FBs is shown in Fig. 1. It is parameterized by $L$ subfilters $Q_{m}(z), L$ delay parameters $N_{m}, L$ lifting coefficients $p_{m}$, and two scaling constants $C_{0}$ and $C_{1}$ for $m=0,1, \ldots, L-1$. It can be seen from Fig. 1 that z-transforms of the analysis and synthesis filters in the lifting structure can be written as follows:

$$
\begin{aligned}
& H_{0}(z)=C_{0} H^{(L-2)}(z), H_{1}(z)=C_{1} H^{(L-1)}(z), \\
& F_{0}(z)=H_{1}(-z) \text { and } F_{1}(z)=-H_{0}(-z) \text {. } \\
& \text { where } H^{(0)}(z)=z^{-2 N_{0}-1}+p_{0} \cdot Q_{0}\left(z^{2}\right) \text {, } \\
& H^{(1)}(z)=z^{-2 N_{1}}+p_{1} \cdot Q_{1}\left(z^{2}\right) H^{(0)}(z) \text {, and } \\
& H^{(m)}(z)=z^{-2 N_{r n}} H^{(m-2)}(z)+p_{m} \cdot Q_{m}\left(z^{2}\right) H^{(m-1)}(z),
\end{aligned}
$$

for $m=2,3, \ldots, L-1$. We shall consider a special case of lifting with identical subfilters:

$$
Q_{L-1}\left(z^{2}\right)=Q_{L-2}\left(z^{2}\right)=\cdots=Q_{1}\left(z^{2}\right)=Q_{0}\left(z^{2}\right)=Q\left(z^{2}\right) .
$$

The delay parameters $N_{m}$ are then given by:

$$
N_{L-1}=\cdots=N_{2}=N_{1}=G \text { and } N_{0}=(G-1) / 2,
$$

where $G$ is the passband group delay of $Q\left(z^{2}\right)$. As a result, the group delays of the analysis filter pair, $H_{0}(z)$ and $H_{1}(z)$, are respectively given by:

$$
G_{0}=(L-1) \cdot G \text { and } G_{1}=L \cdot G
$$

For the simplest case where identical subfilter having the form of $\left(1+z^{-1}\right) / 2$, the $\mathrm{FB}$ is referred to as prototype lifting structured FB. Furthermore, it can be transformed to a new FB using the following substitution of variable: 


$$
x=R_{Q}(\hat{x})=z^{G} Q\left(z^{2}\right),
$$

where $R_{Q}(\hat{x})$ is the zero-phase response of the subfilter $Q\left(z^{2}\right)$ for some positive integer $G$. Since the transformed FB is obtained by replacing $x$ in each lifting step of Fig. 1 by $R_{Q}(\hat{x})$, it can also be implemented by the same number of lifting steps as the prototype.

To analyze the effect of the transformation, let us consider the zero-phase responses of the prototype FB, subfilter and transformed FB in Fig. 2. From (5), the digital frequencies before and after transformation are related by:

$$
x=\cos (\widetilde{\omega})=R_{Q}(\hat{x})=R_{Q}(\cos (\omega)),
$$

where $\omega$ and $\widetilde{\omega}$ are respectively the digital radian frequencies of the prototype and transformed FBs. If $R_{Q}(\hat{x})$ is appropriately designed to have a sharper characteristic than $\hat{x}=\cos \omega$ around $\omega=\pi / 2$, then the transformed $\mathrm{FB}$ will have a much narrower transition band. Furthermore, if we want to preserve the passband and stopband ripples of the prototype FB in the transformed $\mathrm{FB}, R_{Q}(\hat{x})$ should map respectively the values of $x=\cos \widetilde{\omega}$ in the passband and stopband of the prototype FB to the new passband and stopband of the transformed FB. Therefore, the transformed FB can achieve a much narrower and prescribed transition bandwidth by properly designing the subfilter using conventional filter design technique, while preserving the frequency characteristics of the prototype FB.

Using these results, the relations of the prototype $\mathrm{FB}$, subfilter and transformed FB can be summarized as follows:

(i) Zero-phase response of the transformed FB:

$$
\begin{aligned}
& \text { Lowpass: } \begin{cases}1-\delta_{p 0} \leq R_{0}(\hat{x}) \leq 1+\delta_{p 0}, & 0 \leq \omega \leq \omega_{c} \\
\delta_{s 0} \leq R_{0}(\hat{x}) \leq \delta_{s 0}, & \pi-\omega_{c} \leq \omega \leq \pi\end{cases} \\
& \text { Highpass: } \begin{cases}1-\delta_{p 1} \leq R_{1}(\hat{x}) \leq 1+\delta_{p 1}, & \pi-\omega_{c} \leq \omega \leq \pi \\
\delta_{s 1} \leq R_{1}(\hat{x}) \leq \delta_{s 1}, & 0 \leq \omega \leq \omega_{c}\end{cases}
\end{aligned}
$$

where $\omega_{c}$ specifies the cutoff frequency; $\delta_{p i}$ and $\delta_{s i}$ are respectively the passband and stopband ripples of the transformed analysis filters $R_{i}(\hat{x})$, for $i=0,1$.

(ii) Zero-phase response of the prototype FB:

$$
\begin{aligned}
& \text { Lowpass: } \begin{cases}1-\delta_{p 0} \leq \widetilde{R}_{0}(x) \leq 1+\delta_{p 0}, & 0 \leq \widetilde{\omega} \leq \widetilde{\omega}_{c} \\
\delta_{s 0} \leq \widetilde{R}_{0}(x) \leq \delta_{s 0}, & \pi-\widetilde{\omega}_{c} \leq \widetilde{\omega} \leq \pi\end{cases} \\
& \text { Highpass: } \begin{cases}1-\delta_{p 1} \leq \widetilde{R}_{1}(x) \leq 1+\delta_{p 1}, & \pi-\widetilde{\omega}_{c} \leq \widetilde{\omega} \leq \pi \\
\delta_{s 1} \leq \widetilde{R}_{1}(x) \leq \delta_{s 1}, & 0 \leq \widetilde{\omega} \leq \widetilde{\omega}_{c}\end{cases}
\end{aligned}
$$

where $\widetilde{\omega}_{c}$ is the cutoff frequency of the prototype FB.

(iii) Zero-phase response of the subfilter:

$$
\left\{\begin{array}{ll}
\cos \left(\widetilde{\omega}_{c}\right) \leq R_{Q}(\hat{x}) \leq 1, & 0 \leq \omega \leq \omega_{c} \\
-1 \leq R_{Q}(\hat{x}) \leq-\cos \left(\widetilde{\omega}_{c}\right), & \pi-\omega_{c} \leq \omega \leq \pi
\end{array} .\right.
$$

If the prototype FB is monotonic decreasing at the stopband, say a maximally-flat function in 9/7 filter pair [10], then by choosing an appropriate subfilter, an arbitrary small stopband attenuation can be achieved after transformation. Alternatively, a set of prototype PR FBs with different passband/ stopband ripples can be designed offline. The subfilter can then be designed so that the transformed FB will be able to achieve a narrower transition bandwidth, while preserving the ripples of the prototype FB. Optimized prototype FBs usually lead to a better frequency selectivity. It should be noted that this transformation is also valid when $Q(z)$ is a low-delay FIR/IIR function. For more details, interested readers are referred to [6].

\section{Design OF 2D Multi-PLet Filter BANK}

Let $x(\boldsymbol{n})$ be a $N$-dimensional discrete-time signal with $\boldsymbol{n}=\left(n_{0}, \cdots, n_{N-1}\right)^{T}$ and $n_{i} \in \mathcal{N}$, the set of integers. The output $y(\boldsymbol{n})$ of a decimator with an integer sub-sampling matrix $\boldsymbol{M}$ can be written as $y(\boldsymbol{n})=x(\boldsymbol{M n})$. The decimation factor is equal to $|\operatorname{det}(\mathbf{M})|$. Different choices of $\boldsymbol{M}$ give rise to different spectral support of $x(\boldsymbol{n})$ while achieving aliasing-free decimation. The points in $x(\boldsymbol{n})$ that are retained in $y(\boldsymbol{n})=x(\boldsymbol{M n})$ lie on the lattice $\left\{L A T(\boldsymbol{M}): \boldsymbol{t}=\boldsymbol{M n}, \boldsymbol{n} \in \aleph^{* N}\right\}$. Fig. $3 \mathrm{c}$ shows the lattice generated by the $2 \mathrm{D}$ sampling matrix $\boldsymbol{M}_{Q}=\left[\begin{array}{ll}\boldsymbol{M}_{Q_{-} 0} & \boldsymbol{M}_{Q_{-} 1}\end{array}\right]$ associated with the quincunx spectral support in Fig. 3a where $\boldsymbol{M}_{Q_{-}{ }^{0}}=\left[\begin{array}{ll}1 & 1\end{array}\right]^{T}$ and $\boldsymbol{M}_{Q_{-} 1}=\left[\begin{array}{ll}1 & -1\end{array}\right]^{T}$. It can also be seen that those points on $\operatorname{LAT}(\boldsymbol{M})$ are in fact generated by the linear combinations of $\boldsymbol{M}_{Q_{-} 0}$ and $\boldsymbol{M}_{Q_{-} 1}$.

Fig. 4 shows the general structure of a two-channel critically decimated $N$-D FB with sub-sampling matrix $\boldsymbol{M}$ and $|\operatorname{det}(\boldsymbol{M})|=2$. The analysis and synthesis filters are expressed respectively in their type-I and type-II polyphase representations, where $\boldsymbol{E}(\boldsymbol{z})$ and $\boldsymbol{R}(\boldsymbol{z})$ are their polyphase matrices. $\boldsymbol{k}_{0}$ and $\boldsymbol{k}_{1}$ belong to the set of integer vectors, $\boldsymbol{\aleph}(\boldsymbol{M})$, which lie inside the fundamental parallelepiped of $\boldsymbol{M}$, $F P D(\boldsymbol{M})$, where $F P D(\boldsymbol{M})$ is the region spanned by $\boldsymbol{M} \cdot \boldsymbol{x}$, with $x_{i} \in[0,1), i=0, \ldots, N-1$. The gray parallelogram area in Fig. 3c spanned by the two vectors $\left(\boldsymbol{M}_{Q_{-} 0}, \boldsymbol{M}_{Q_{-} 1}\right)$ is the $F P D(\boldsymbol{M})$ associated with $\boldsymbol{M}_{Q}$. If the product $\boldsymbol{R}\left(\boldsymbol{z}^{M}\right) \boldsymbol{E}\left(\boldsymbol{z}^{M}\right)$ is equal to a constant multiplies of signal delay, then the system is PR.

Similar to [1], a simple method for achieving this condition can be done by transformation of 1D PR FB. More precisely, $Q\left(z^{2}\right)$ is transformed to $Q\left(z^{M_{0}}\right) Q\left(z^{M_{1}}\right)$ and the 1-D delay $z^{2}$ is replaced with $z^{M_{0}} z^{M_{1}}$ in Fig. 1 . Then, the $2 \mathrm{D}$ analysis filters can be written as follows:

$$
H_{0}(\boldsymbol{z})=C_{0} H^{(L-2)}(\boldsymbol{z}) \text { and } H_{1}(\boldsymbol{z})=C_{1} H^{(L-1)}(\boldsymbol{z})
$$

where $H^{(0)}(z)=z^{-k_{t}}\left(z^{M_{0}} z^{M_{t}}\right)^{-N_{0}}+p_{0} \cdot Q\left(z^{M_{0}}\right) Q\left(z^{M_{1}}\right)$,

$H^{(1)}(\boldsymbol{z})=\left(\boldsymbol{z}^{\boldsymbol{M}_{0}} \boldsymbol{z}^{\boldsymbol{M}_{I}}\right)^{-N_{1}}+p_{1} \cdot Q\left(\boldsymbol{z}^{\boldsymbol{M}_{0}}\right) Q\left(\boldsymbol{z}^{\boldsymbol{M}_{1}}\right) H^{(0)}(\boldsymbol{z})$, and

$H^{(m)}(\boldsymbol{z})=\left(\boldsymbol{z}^{M_{0}} z^{M_{\boldsymbol{I}}}\right)^{-N_{m_{2}}} H^{(m-2)}(\boldsymbol{z})+p_{m} Q\left(\boldsymbol{z}^{M_{0}}\right) Q\left(\boldsymbol{z}^{M_{1}}\right) H^{(m-1)}(\boldsymbol{z})$, for $m=2,3, \ldots, L-1$. Hence, the problem remaining is to determine the sampling matrix $\boldsymbol{M}$ and the integer vectors $\boldsymbol{k}_{0}$ and $\boldsymbol{k}_{1}$ according to the desired spectral support. Suppose $x(\boldsymbol{n})$ is decimated by $\boldsymbol{M}$, its aliasing-free spectral support should be equal to the spectral support $\Omega$ of the analysis lowpass filter, which is given by $\left\{\Omega: \boldsymbol{\omega}=\pi \boldsymbol{M}^{-T} \boldsymbol{x}, \boldsymbol{x} \in[-1,1)^{2}\right\}$ [14]. For notational convenience, let $\pi \boldsymbol{M}^{-T}=\left[\boldsymbol{\omega}_{0}, \boldsymbol{\omega}_{1}\right]$. In general, if $\left[\omega_{0}, \omega_{1}\right]$ can be determined from the desired spectral support, then $\boldsymbol{M}$ can be computed from $\boldsymbol{M}=\left[\begin{array}{ll}\boldsymbol{\omega}_{0} & \boldsymbol{\omega}_{1}\end{array}\right]^{-T} / \pi$. Consider the quincunx sampling as an example, the spectral support is a parallelogram defined by the two vectors, $\boldsymbol{\omega}_{0}=\left[\begin{array}{ll}\pi / 2 & \pi / 2\end{array}\right]^{T}$ and $\boldsymbol{\omega}_{1}=\left[\begin{array}{ll}-\pi / 2 & \pi / 2\end{array}\right]^{T}$ as shown in Fig. 3a. Using this result, it can easily be verified that the sampling 
matrix of the quincunx spectral support is given by $\boldsymbol{M}_{Q}=\left[\begin{array}{ll}\boldsymbol{M}_{Q_{-} 0} & \boldsymbol{M}_{Q_{-} 1}\end{array}\right]$, where $\quad \boldsymbol{M}_{Q_{-} 0}=\left[\begin{array}{ll}1 & 1\end{array}\right]^{T}$ and $\boldsymbol{M}_{Q_{-} 1}=\left[\begin{array}{ll}1 & -1\end{array}\right]^{T}$, and the corresponding values of $\boldsymbol{k}_{0}$ and $\boldsymbol{k}_{1}$ are respectively seen to be $\boldsymbol{k}_{0}=\left[\begin{array}{ll}0 & 0\end{array}\right]^{T}$ and $\boldsymbol{k}_{1}=\left[\begin{array}{ll}1 & 0\end{array}\right]^{T}$. Substituting $z^{M_{Q_{-} 0}}=z_{0} z_{1}, \quad z^{M_{Q_{-}}}=z_{0} z_{1}^{-1}, \quad z^{M_{Q}}=z_{0}^{2}$, and $z^{k_{1}}=z_{0}$ into (9) gives the frequency response of the analysis filters. To design a PR FB with a hourglass spectral support, we can multiply $x(\boldsymbol{n})$ and $\hat{x}(\boldsymbol{n})$ in the quincunx PR FB by $(-1)^{m_{1}}$ [13]. The calculation of parallelogram support is similar. It can be seen from Fig. $3 \mathrm{~b}$ that $\boldsymbol{\omega}_{0}=\left[\begin{array}{ll}\pi / 2 & 0\end{array}\right]^{T}$ and $\boldsymbol{\omega}_{1}=\left[\begin{array}{ll}-\pi / 2 & \pi\end{array}\right]^{T}$. Therefore, $\boldsymbol{M}_{P}$ is given by $\boldsymbol{M}_{P}=\left[\begin{array}{ll}\boldsymbol{M}_{P_{-} 0} & \boldsymbol{M}_{P_{-} 1}\end{array}\right]$, where $\boldsymbol{M}_{P_{-} 0}=\left[\begin{array}{ll}2 & -1\end{array}\right]^{T}$ and $\boldsymbol{M}_{P_{-} 1}=\left[\begin{array}{ll}0 & 1\end{array}\right]^{T}$, and $\boldsymbol{k}_{0}$ and $\boldsymbol{k}_{1}$ are determined to be $\boldsymbol{k}_{0}=\left[\begin{array}{ll}0 & 0\end{array}\right]^{T}$ and $\boldsymbol{k}_{1}=\left[\begin{array}{ll}1 & 0\end{array}\right]^{T}$, respectively.

\section{Design Examples}

\section{Example 1: 1D and 2D Transformed (9,7) Filter Banks}

In this example, the $(9,7) \mathrm{FB}$ is used as the prototype $\mathrm{FB}$. The corresponding lifting coefficients and scaling constants are listed in the second column of table 1. Fig. 5a shows the frequency responses of the transformed $(9,7)$ FBs with different transition bandwidth. This demonstrates the flexibility of the proposed approach in controlling the frequency characteristics of the PR FBs. Similar argument holds for the 2D case. It can be seen from Figs. $5 b-5 d$ that the $2 D$ quincunx multi-plet FBs have different transition bandwidths according to the cutoff frequencies of the subfilter. Furthermore, if the subfilter is realized as variable digital filters [15], the frequency characteristics of the transformed multi-plet FBs can be tuned online. This offers us more flexibility in processing $2 \mathrm{D}$ signals.

\section{Example 2: Low-delay 2D multi-plet Filter Banks}

In this example, the 1-D low-delay multi-plet PR FB designed in Example 2 in [6] is considered. The maximum passband and stopband ripples are about $3.45 \times 10^{-5}$ and $3.16 \times$ $10^{-3}$, respectively. The group delays of the analysis lowpass and highpass filters are respectively 63 and 84 samples, as compared to 93 and 124 samples for the linear-phase case. The frequency and group delay responses of the 1D multi-plet FB are respectively shown in Figs. $6 \mathrm{a}$ and $6 \mathrm{~b}$. As described in Section III, it can be transformed to obtain the low-delay 2D multi-plet FBs with various spectral supports. Figs. $6 \mathrm{c}$ and $6 \mathrm{~d}$ illustrate the effectiveness of the proposed approach in designing low-delay 2D multi-plet PR FB with hourglass and parallelogram spectral supports. The former is obtained by shifting the frequency spectrum of the quincunx FB via the modulation $e^{j \pi n_{1}}$, where $n_{1}$ is the integer index in the vertical direction. Moreover, by properly cascading the 2D PR FBs with either quincunx or hourglass spectral supports in a tree structure, as suggested in [12], it is possible to realize the multiplet PR directional FBs. Due to page limitation, Figs. 6e and $6 \mathrm{f}$ only show the channels 0 and 3 of that class of directional FB. Interested readers are referred to [12] for more details on the desired spectral support.

\section{Conclusion}

The design of two-channel 2D multi-plet nonseparable PR FBs is presented. By employing a $1 \mathrm{D}$ to $2 \mathrm{D}$ mapping, 2D PR
FBs with quincunx, hourglass, and parallelogram spectral support can be obtained. Thanks to the frequency transformation technique, the characteristics of these nonseparable FBs can be varied on-line and they offer more flexibility in processing 2D signals. By cascading multiple multi-plet FBs with appropriate spectral support, PR directional FBs with variable characteristics can readily be obtained.

\section{REFERENCES}

[1] S. M. Phoong, C. W. Kim, P.P. Vaidyanathan and R. Ansari, "A new class of two-channel biothogonal filter banks and wavelet bases," IEEE Trans. Signal Processing, vol. 43, pp. 649-664, March 1995.

[2] R. Ansari, C. W. Kim and M. Dedovic, "Structure and design of two-channel filter banks derived from a triplet of halfband filters," IEEE Trans. Circuits Syst. II, vol. 46, no. 12, pp. 1487 $1496,1999$.

[3] D. B. H. Tay, "A novel approach to the design of the class of triplet halfband filterbanks," IEEE Trans. Circuits Syst. II, vol. 57, issue 7, pp. 378-383, July 2004.

[4] K. S. Yeung and S. C. Chan, "On the Design and Multiplier-less Realization of Perfect Reconstruction Triplet-based FIR Filter Banks and Wavelet Bases," IEEE Trans. Circuits Syst. I, vol. 51, pp. 1476-1491, Aug. 2004.

[5] I. Daubechies and W. Sweldens, "Factoring wavelet transform into lifting steps," J. Fourier Anal. Appl., vol. 4, no. 3, pp. 247269, 1998.

[6] S. C. Chan and K. M. Tsui, "Multi-plet two-channel perfect reconstruction filter banks," in Proc. IEEE ISCAS'2005, pp. 4297-4300, May 23-26, 2005.

[7] T. Saramaki, "Design of FIR filters as a tapped cascaded interconnection of identical subfilters," IEEE Trans. Circuits Syst., vol. 34, pp. 1011-1029, Sept. 1987.

[8] D. B. H. Tay and N. G. Kingsbury, "Flexible Design of Multidimensional Perfect Reconstruction FIR 2-Band Filters using Transformations of Variables," IEEE Trans. Image Processing, vol. 2, no. 4, pp. 466-480, October 1993.

[9] T. G. Marshall, Jr., "Zero-phase filter bank and wavelet coder matrices: properties, triangular decompositions, and a fast algorithm" Multidimensional Syst. Signal Processing: Special Issue on Wavelets and Multiresolution Signal Processing, vol. 7 , 1996.

[10] A. Cohen, I. Daubechies and J. C. Feauveau, "Biorthogonal bases of compactly supported wavelets," Communications on Pure and Appl. Math., vol. 45, pp. 485-560, June 1992.

[11] S. C. Chan, K. S. Pun and K. L. Ho, "On the design and implementation of a class of multiplier-less two-channel 1-D and 2-D nonseparable PR FIR filterbanks" in Proc. IEEE ICIP, vol. 2, pp. 241-244, Oct. 2001.

[12] T. T. Nguyen and S. Oraintara, "A Class of Directional Filter Banks," in Proc. IEEE ISCAS'2005, pp. 1110-1113, May 2005.

[13] R. H. Bamberger, and M. J. T Smith, "A filter bank for the directional decomposition of images: theory and design", IEEE Trans. SP., vol. 40. No.4, pp. 882-893, April 1992.

[14] P. P. Vaidyanathan, "Multirate systems and filter banks", Prentice Hall, 1993.

[15] K. S. Pun, S. C. Chan, K. S. Yeung and K. L. Ho, "On the Design and Implementation of FIR and IIR Digital Filters with Variable Frequency Characteristics," IEEE Trans. Circuits Syst. II, vol. 49, no. 11, pp. 689-703, Nov, 2002.

\begin{tabular}{||c|c|c||}
\hline & (9,7) filter pair [10] & Prototype FB [6] \\
\hline$p_{0}$ & $-\mathbf{3 . 1 7 2 2 6 8 6 8 4 0 8 2 8 9}$ & 0.23987556667257 \\
\hline$p_{1}$ & -0.10596023714940 & -0.54571527976115 \\
\hline$p_{2}$ & 1.76582215102264 & 0.54045911345798 \\
\hline$p_{3}$ & 0.88701370409649 & -0.23167459250035 \\
\hline$C_{0}$ & 0.81289306611600 & 0.71401331291255 \\
\hline$C_{1}$ & 0.61508705245638 & 0.71237102180672 \\
\hline
\end{tabular}

Table 1. Lifting coefficients and scaling constants of the prototype FBs. 


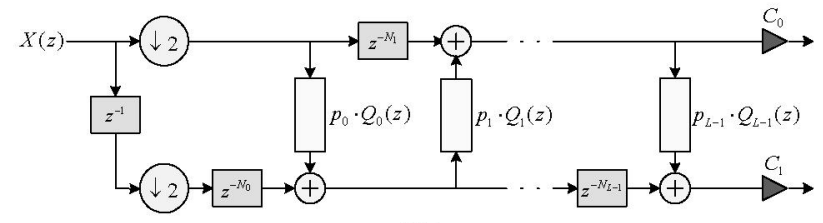

1(a)



1(b)

Fig. 1: Structure of the multi-plet FBs: (a) Analysis bank. (b) Synthesis bank.

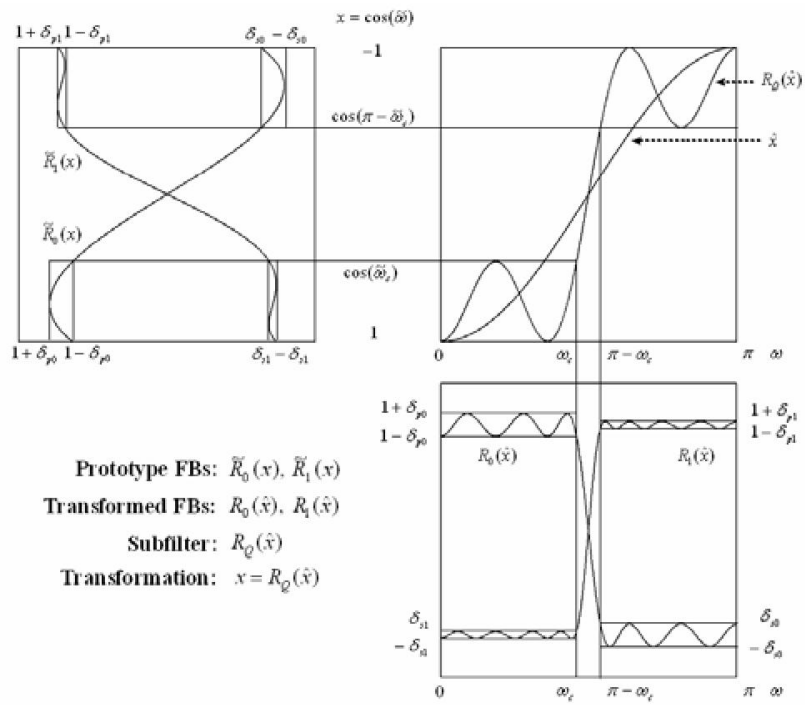

Fig. 2: General relations and specifications of the prototype FB, the subfilter and the multi-plet transformed FB.

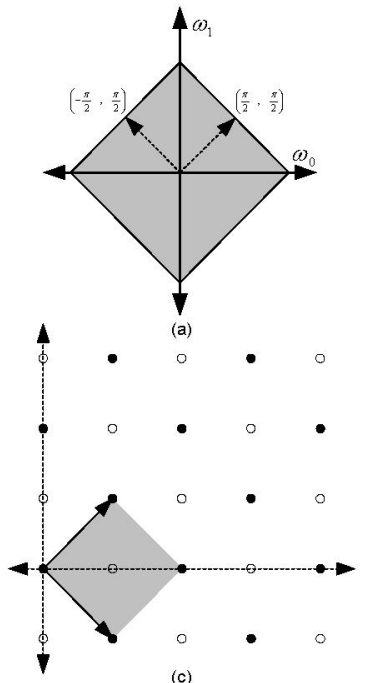

$M=\left(\begin{array}{cc}1 & 1 \\ 1 & -1\end{array}\right), k_{0}=\left[\begin{array}{l}0 \\ 0\end{array}\right], k_{1}=\left[\begin{array}{l}1 \\ 0\end{array}\right]$

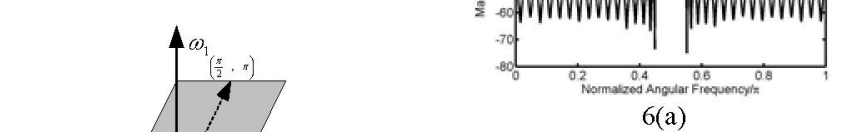

6(a)

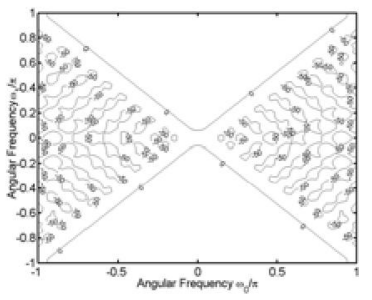

$6(\mathrm{c})$

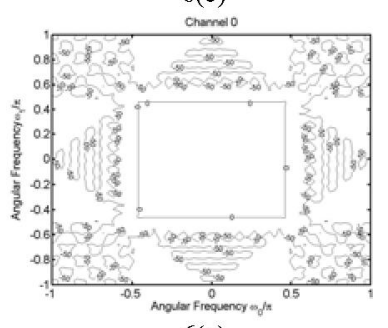

6(e)

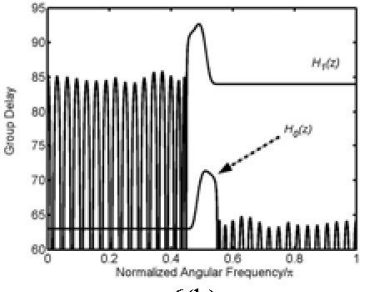

6(b)

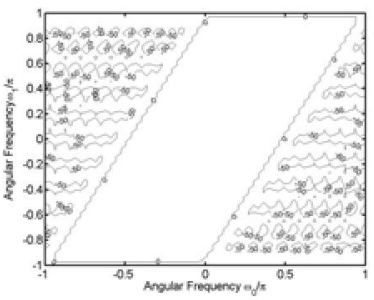

$6(d)$

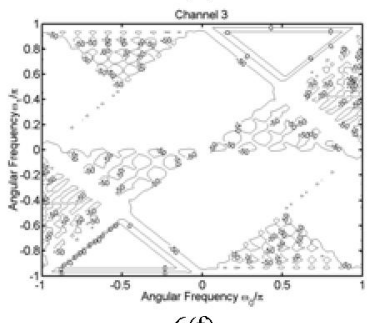

$6(f)$
Fig. 3: (a) Spectral support of quincunx sampling, (b) spectral support for the parallelogram filter, (c) sampling matrix and its associated lattice for quincunx sampling, (d) sampling matrix and its associated lattice for the parallelogram filter.
Fig. 6. (a) Frequency response and (b) group delay response of $1 \mathrm{D}$ lowdelay multi-plet FB. Frequency responses of $2 \mathrm{D}$ nonseparable PR FB in example 2: (c) Hourglass (d) Parallelogram. (e) Channel 0 and (f) channel 3 of directional FB in [12]. 